\title{
CLINICAL PROFILE OF LINEAR DERMATOSES EMPHASIZING ON THEIR PRESENTATION ALONG THE BLASHCO'S LINES
}

\author{
N. Ramanjanamma ${ }^{1}$, Garnipudi Pavani *2, Jasmine D ${ }^{2}$. \\ ${ }^{1}$ Venereology, Associate professor of Dermatology, Viswabharathi Medical College, Kurnool. \\ ${ }^{2}$ Assistant professor of DVL, Viswabharathi Medical College, Kurnool. \\ ABSTRACT
}

Introduction: Skin lesions present with innumerable patterns like Discoid, Petaloid, Arcuate, Annular, Polycyclic, Livedo, Reticulate, Target, Stellate, Digitate, Linear, Serpiginous and Whorled. Most of the linear lesions follow the Blaschko's lines. In this study the incidence of linear dermatoses, the age and sex incidence, various types of clinical presentation among the patients, the association, and various sites of distribution and clinic histopathological correlation of various linear dermatoses.

Materials and Methods: A Descriptive study of patients with linear dermatoses attending the Outpatient Department of Viswabharathi Medical College, Kurnool, from January 2017 to December 2017. Detailed history including family history, Marital Status, History of trauma documented with clinical examination. After counselling and after recording their consent for the test, skin biopsy done along with routine investigations and the results are evaluated.

Results: Among the 90 cases, Lichen striatus was the most common presentation followed by linear epidermal naevus and Linear Lichen planus in our study. Family history of similar leisions in any of these patients. Out of 90 cases, 83 cases showed unilateral distribution and only the remaining 7 showed bilateral distribution of lesions in a linear pattern. 66 cases, had lesions mainly over the extremities, corresponding to the lines of Blaschko.

Conclusion: The Incidence of Linear Dermatoses during the period of January 2017 to December 2017 was $0.2 \%$ Lichen striatus was founded more common, female preponderance. Majority of patients showed unilateral distribution more on the extremities. Histopathological correlation shows the importance of histopathology which ultimately changing the management in any given condition. Very few associations were noted.

KEY WORDS: Linear Dermatoses, lines of Blaschko, skin biopsy, Lichen striatus, and Distribution of lesions in a linear pattern.

Address for correspondence: Dr. Garnipudi Pavani, Assistant professor of DVL, Viswabharathi Medical College, Kurnool, Andhra Pradesh, India. E-Mail: pavanigarnipudi@gmail.com

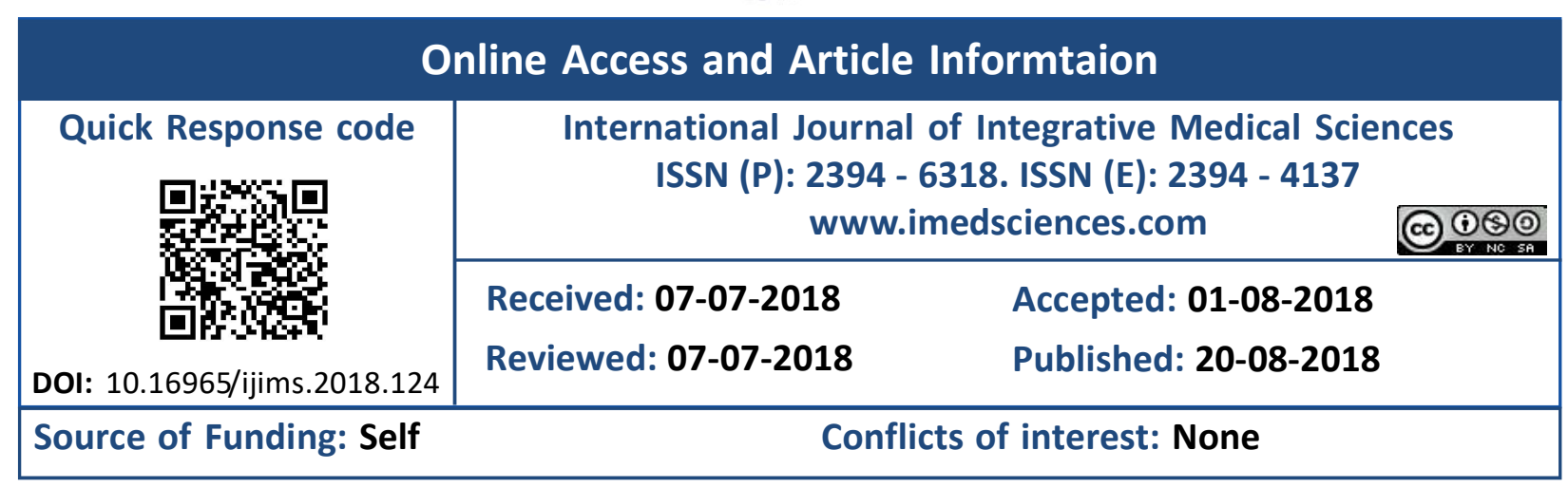

\section{BACKGROUND}

Dermatology is a visual specialty and many skin lesions may be diagnosed at a glance. The morphology of individual lesions, their overall pattern, their special relationship to each other and their site of distribution in the body are helpful and provide an easily recognizable clue to a rapid visual diagnosis. Skin lesions present with innumerable patterns like linear, arcuate, annular, reticulate, serpinginous, discoid etc. Linear configuration due to course of blood vessel, nerve and lymphatic, dermatomal involvement, developmental causes, and external factors such as plants, physical factors (koebner's phenomenon), chemicals, allergens and thermal these are responsible for linear skin lesions. 
It is speculated that linear dermatoses following Blaschko's line suggest that the tendency for development of a particular dermatoses is predetermined during embryogenesis. Exposure to an appropriate trigger such as an autoimmune attack may result in the development of linear dermatoses [1]. Most of the linear lesions follow the Blaschko's lines are characteristic of mosaic conditions of the epidermis [2,3]. They were defined by Dr. Alfred Blaschko, a dermatologist from Berlin, who drew the common linear distribution patterns of numerous nevus cases he had seen. They are believed to represent the lines of migration of embryonal cells proliferating from the neural crest and are also adopted by abnormal (mutated) cells resulting in the linear appearance of the lesions. Blaschko's lines are the pattern assumed by many congenital as well as acquired disorders. They do not follow any known nervous, vascular or lymphatic distribution [4]. These are convexly arch-shaped at the chest S-shaped on the abdomen, V-shaped on the central back and slightly curved straight line on the extremities. On the forehead the lines of Blaschko run in a funnellike configuration from scalp to the eyebrow and converge on the nasal root. From the nasal root they run in four main directions. In the first direction they follow a perpendicular direction to the tip of the nose [5]. Several skin diseases are distributed along Blaschko's lines including various forms of epidermal and adnexal nevi, inflammatory dermatoses (such as Lichen striatus) and genodermatoses expressed in mosaic form (such as Epidermolytic hyperkeratosis, Ito's hypomelanosis, Incontinentia pigmenti, Mibelli's porokeratosis, Leiomyomas) [5]. As there is paucity of studies on this subject, a clinical study of linear dermatoses with special emphasis on their presentation along the Blaschko's line was carried out.

\section{MATERIALS AND METHODS}

A Descriptive study of patients with linear dermatoses attending the Outpatient Department of Viswabharathi Medical College, Kurnool, from January 2017 to December 2017, patients presenting with cutaneous lesions in a linear pattern and along the Blaschko's lines were included in the study after taking the consent from, and linear cutaneous lesions secondary to infections and contact dermatitis were not included in the study. The patient or from the parent/guardian (in case the patient is a minor), the detailed history, including the family history and history of consanguinity were obtained to rule out genetic inheritance. Complete cutaneous and systemic examinations were done. Relevant investigations were performed wherever necessary. Wood's lamp examination and digital photography of the dermatoses were taken. Skin biopsy for histopathology was done and special stains were used wherever necessary. Patients presenting with cutaneous lesions in a linear pattern and along the Blaschko's lines were included, and linear cutaneous lesions secondary to infections and contact dermatitis were not involved the study.

\section{RESULTS}

Among the study group of 90 cases, 68 cases were asymptomatic and reported for cosmetic reasons. Intense itching was the main reason to bring the lichen planus patients and few cases of the lichen striatus patients for treatment.

Table 1: list of va rious linear dermatoses.

\begin{tabular}{|c|c|c|}
\hline Lichen Striatus & 25 & 27.78 \\
\hline Linear epidermal Nevus & 19 & 21.11 \\
\hline Linear Lichen Planus & 18 & 20 \\
\hline Linear Morphoea & 9 & 10 \\
\hline ILVEN & 3 & 3.33 \\
\hline Segmental Vitiligo & 3 & 3.33 \\
\hline Hypomelanosis of Ito & 3 & 3.33 \\
\hline Linear lichenin dermatitis & 2 & 2.22 \\
\hline Linear psoriasis & 2 & 2.22 \\
\hline Segmental & 2 & 2.22 \\
\hline Neurofibromatosis & 1 & 1.11 \\
\hline LWNH & 1 & 1.11 \\
\hline Incontinentia pigmenti & 1 & 1.11 \\
\hline Nevus depigmentosus & 1 & 1.11 \\
\hline Linear porokeratosis & 90 & 100 \\
\hline Total &
\end{tabular}

Table 2: Age and Sex distribution in this study of 90 patients with Linear lesions.

\begin{tabular}{|c|c|c|c|}
\hline AGE & Male & Female & Total \\
\hline $\mathbf{0 1 - 1 0}$ & 9 & 14 & 23 \\
\hline $\mathbf{1 1 - 2 0}$ & 6 & 13 & 29 \\
\hline $\mathbf{2 1 - 3 0}$ & 13 & 5 & 18 \\
\hline $\mathbf{3 1 - 4 0}$ & 1 & 4 & 5 \\
\hline $\mathbf{4 1 - 5 0}$ & 3 & 8 & 11 \\
\hline $\mathbf{5 0}$ & 2 & 2 & 4 \\
\hline Total & 44 & 46 & 90 \\
\hline
\end{tabular}


Lichen Striatus: Among the 90 patients in this group 25 presented with Lichen striatus in the age group between 11 months and 45 years and forming male to female ratio of 11:14.

Table 3: Age and Sex distribution among patients with Lichen striatus $(\mathrm{N}=25)$.

\begin{tabular}{|c|c|c|c|}
\hline AGE & Male & Female & Total \\
\hline $\mathbf{0 1 - 1 0}$ & 4 & 4 & 8 \\
\hline $\mathbf{1 1 - 2 0}$ & 4 & 4 & 8 \\
\hline $\mathbf{2 1 - 3 0}$ & 3 & 2 & 5 \\
\hline $\mathbf{3 1 - 4 0}$ & 0 & 2 & 2 \\
\hline $\mathbf{4 1 - 5 0}$ & 0 & 2 & 2 \\
\hline$>\mathbf{5 0}$ & 0 & 0 & 0 \\
\hline Total & 11 & 14 & $\mathbf{2 5}$ \\
\hline
\end{tabular}

Table 4: Sites of Distribution of Lichen Striatus.

\begin{tabular}{|c|c|}
\hline Sites of Distribution & No. of Cases \\
\hline Upper limb & 8 \\
\hline Lower limb & 7 \\
\hline Trunk & 4 \\
\hline Trunk \& Upper limb & 2 \\
\hline Trunk \& Lower limb & 2 \\
\hline Trunk, Upper \& Lower limb & 1 \\
\hline Neck & 1 \\
\hline Total & 25 \\
\hline
\end{tabular}

Linear Lichen Planus: In this study group 18 patients presented with linear lichen planus. The age group ranged between 7 years and 70 years with an average of 30 years. Out of the 18 cases 11 cases were males and 7 cases were females forming a male: female ratio of 1.5:1.

Table 5: Age and Sex distribution among patients with Lichen Planus ( $n-18)$.

\begin{tabular}{|c|c|c|}
\hline Sites of Distribution & Right & Left \\
\hline Upper limb & 1 & 1 \\
\hline Lower limb & 5 & 7 \\
\hline Trunk & 1 & 0 \\
\hline Head \& Neck & 0 & 1 \\
\hline Upper limb \& Lower limb & 1 & 0 \\
\hline Lower limb \& Trunk & 1 & 0 \\
\hline Total & 9 & 9 \\
\hline
\end{tabular}

Table 6: Distribution of Linear Epidermal Verrucous Nevus (n-19).

\begin{tabular}{|c|c|}
\hline SITES & No. of cases \\
\hline Head \& Neck & 6 \\
\hline Head, Neck \& Trunk & 3 \\
\hline Lower limb \& Trunk & 3 \\
\hline Upper limb \& Trunk & 1 \\
\hline Upper \& Lower limb, Trunk & 1 \\
\hline Trunk & 1 \\
\hline Right upper limb & 1 \\
\hline Left upper limb & 1 \\
\hline Right lower limb & 1 \\
\hline Left lower limb & 1 \\
\hline Total & 19 \\
\hline
\end{tabular}

Int J Intg Med Sci 2018;5(7):687-92. ISSN 2394 - 4137
Linear Morphoea: In this study group 9 patients of linear morphoea was recorded. Two cases were linear pansclerotic morphea, 3 cases were En-coup-de sabre, 3 cases are linear morphoea in extremities and trunk, finally one case was PARRY ROMBERG SYNDROME.

Table 7: Age and Sex Distribution of Linear Morphoea (n-9).

\begin{tabular}{|c|c|c|c|}
\hline AGE & Male & Female & Total \\
\hline $\mathbf{0 1 - 1 0}$ & 0 & 4 & 4 \\
\hline $\mathbf{1 1 - 2 0}$ & 0 & 3 & 3 \\
\hline $\mathbf{2 1 - 3 0}$ & 1 & 1 & 2 \\
\hline Total & 1 & 8 & 9 \\
\hline
\end{tabular}

Table 8: Site of Distribution of Linear Morphoea (n -9).

\begin{tabular}{|c|c|}
\hline Sites of Distribution & No. of Cases \\
\hline Upper limb & 1 \\
\hline Lower limb & 2 \\
\hline Upper limb \& Lower limb & 1 \\
\hline Head \& Neck & 4 \\
\hline Trunk, Upper \& Lower limb & 1 \\
\hline Total & 9 \\
\hline
\end{tabular}

\section{DISCUSSION}

In this study of 90 cases with a linear distribution, of the lesions which did not exhibit Koebner's phenomenon, none of the cases seemed to follow the linearity determined by the Nerves, vascular or lymphatic structure and it has been suggested that these lesions develop in the Lines of Blaschko. Hence the various nevoid and acquired conditions which are supposed to follow the Lines of Blaschko, which are thought to be due to a form of human mosaicism were included in this study. Most of the patients were asymptomatic and mainly came for cosmetic reasons, except the linear lichen planus patients and few of Lichen striatus patients who had presented in our Dermatology Outpatient Department for intense itching.

Lichen striatus formed the majority of cases amounting to 25 in this study. The condition is said to occur commonly in the age group of 5 - 15 years whereas in the present study, majority of patients were in the age group of between 3-20 years. It was more commonly observed in females in this study group with a Male: Female ratio $11: 14$ as also been documented by Hauber et al. (6). The lesions are normally asymptomatic, with occasional 
pruritus in the study group as described in the literature. There were no predisposing factors in any of the patients, as Lichen striatus is of unknown etiology. Most of the patients in the study group had lesions over the extremities, but few patients had lesions also over the trunk as recorded in the literature, which complied with the variable sites of expression. All the patients had unilateral distribution of the lesions. Two of the patients showed nail changes among this group, although changes in the form of longitudinal ridging, subungual hyperkeratosis, splitting and onycholysis have been documented. Atopy was found to be associated with Lichen striatus in $80 \%$ of patients, although none in this study group had personal or family history of atopy but associated lesions like pityriasis veriscolor, xerosis and photosensitivity were seen, which were not documented so far and may be coincidental. Histopathological examination showed a chronic dermatitis picture in majority of patients in this study group, few cases showed psoriasi form dermatitis like features and some other showed lichenoid dermatitis like picture which was consistent with the variable histological pictures as described in the literature. There were no systemic abnormalities noted in any of the Lichen striatus patients in this study group.

Linear lichen planus formed the next common condition in this study group consisting of 18 cases. Among them most of the patients were in the age group between $11-30$ years and the average age at the time of diagnosis was 25 years. In this study Male: Female ratio of 11:7 was noted, showing a slight male preponderance in contrary to the literature [7].

There was no history of contact with any chemicals or trauma but 2 patients had history of intake of NSAID and another 2 were incidentally associated with freckle and Becker's naevus. There was no history of similar lesions in their family members or any other associated autoimmune disorders. It has been suggested that the linear distribution seen could be due to the tendency of Lichen Planus to develop, with the formation of clone of predisposed or vulnerable cells, which is predetermined during embryogenesis. Most of the patients presented with the complaints of intense itching. These lesions started as hyper pigmented, discrete, flat topped papules with a violaceous hue distributed in a linear pattern. In some of the patients, the papules coalesced to form the linear plaques which were continuous or interrupted. Among 18 patients 2 patients had nail changes and one patient had mucous membrane involvement. In sixteen patients, the lesions were found on the extremities. Two patients showed multiple linear lesions following the lines of Blaschko, but no immune compromised state was noted as shown in literature. The length of the lesions ranged from $5 \mathrm{cms}-50 \mathrm{cms}$ and 4 patients had lesions extending the entire length of the limb. On histopathological examination, sixteen out of 18 lesions showed the classical features of Lichen planus as described in the literature [8].

One picture showed normal epidermis, basal cell degeneration, superficial mononuclear cell infiltrate in upper dermis, colloid bodies and pigment incontinence which was fit into the features of Lichenoid dermatitis. One case was diagnosed clinically as lichen planus but HPE showed features of Lichen planus pigmentosus (LPP) usually manifests: In contrast to lichen planus it is asymptomatic dark brown patches, with longer clinical course, usually occurs in sun exposed site-face, neck and upper trunk, and striking predominance of LPP in flexures -axilla and inguinal region. But in this case it has unusual presentation like unilateral distribution, linear pattern, and Lesions in unexposed areas. HbsAg seropositivity was found in one of the patient, which has not been reported in literature. Some of the associated features were Becker's nevus, pityriasis versicolor, Tinea corporis, varicose vein, chronic urticaria, freckle which may be coincidental.

In this study, linear epidermal nevus accounted for 19 cases, with 13 cases between the age group of 1 and 20 years. Out of whom 12 were males and 7 were females. A male preponderance was seen in this study, in contrary to equal sex incidence given in the literature [9]. All the patients (19) with this disorder mainly came for cosmetic reason. No family history of similar lesion was recorded. The lesions manifested as hyper pigmented verrucous papules arranged in a linear continuous or interrupted bands and majority of our patient the lesions were since 
birth, but in few of the patients the lesions became apparent later in life. In this age group 5 patients had lesions involving trunk and extremities, implicating systemized form of verrucous epidermal nevus. Most (17 out of 19) of the skin biopsies showed the classical features of verrucous epidermal nevus like hyperkeratosis, irregular acanthosis, papillomatosis, well formed granular layer, increased pigment basal layer and patchy inflammatory infiltrates in upper dermis. Two of them showed the features of Inflammatory Linear verrucous epidermal nevus like hyperkeratosis with foci parakeratosis, acanthosis, elongation and thickening of the rete ridges with a 'psoriasiform' appearance, papillomatosis, and spongiosis with exocytosis of lymphocytes.

In this study nine cases of Linear Morphoea were recorded, among them 7 were less than 20 years of age. Generally the peak incidence of this condition is between 20 and 30 years of the age group [9]. Out of the 9 cases, 1 was male and 8 were female patients forming a Male: Female ratio 1:8 correlating with the female preponderance of this condition as recorded in the literature [9]. Except one who had prior history of intramuscular injection over the lesional site, In all others [8] there was no history of any provocative factors like trauma or drug intake prior to the onset of lesion. There was no history of similar lesion in the family members. Most of the patients with an asymptomatic atrophic plaques except one who had pain over the plaque. Most of the patients (4 out of 9 ) had lesions over the head and neck region among this one case was diagnosed as Parry Romberg syndrome and 5 patients had lesions over the lower limb, which is the commonest site of involvement shown in literature [9]. All the investigations pertaining to Morphoea were found to be normal including $X$ - ray, except 4 patients who had eosinophilia and 3 cases that showed ANA positivity. Most (6 out of 9) of the skin biopsies showed the classical features of morphoea.

In this study, among the two patients, one had presented with hypo pigmented scaly plaque of 6 months duration over the extremity. There was no evidence of any trauma preceding the lesion. The patient had fine, regular pitting over the finger nails. On histopathological examination the lesion showed a characteristic feature of psoriasis, with which, the clinical diagnosis was revised from lichen striatus to linear psoriasis. Second patient was presented with asymptomatic, erythematous plaques over the extremity on histopathological examination, it was found to have hypergranulosis and orthokeratosis, alternating with absent granular layer and parakeratosis and had other characteristic features of psoriasis.

In this study group, three ( 2 female +1 male) patients presented with segmental vitiligo between the age group of 8 years and 21 years which was earlier than the other types of vitiligo. The lesions manifested as patches, arranged in a linear, continuous or interrupted bands, involving mainly the extremities. One had lesions over the face and neck region corresponding to the dermatomes rather than Blaschko's lines, perhaps in keeping with a neuronal etiological theory of vitiligo and it could be also clonal susceptibility of melanocytes to neurons. One patient had leukotrichia over the vitiligo patches. There was no family history of similar lesions or any mucosal or nail involvement. None of them had vitiligo elsewhere in the body. There was no other associated auto immune disorders. Biopsy of these lesions showed the features consistent with vitiligo, like flaky hyperkeratosis, normal epidermis and absence of melanocytes in the basal cell layer and sparse inflammatory in upper dermis.

\section{CONCLUSION}

The Incidence of Linear Dermatoses during the period of January 2017 to December 2017 was $0.2 \%$ Lichen striatus was founded more common, female preponderance. Majority of patients showed unilateral distribution more on the extremities. Histopathological correlation shows the importance of histopathology which ultimately changing the management in any given condition. Very few associations were noted.

\section{REFERENCES}

[1]. Soma Y, Fujimoto M. Frontoparietal scleroderma (en coup de sabre) following Blaschko's lines. J Am Acad Dermatol 1998; 38: 366-8. 
[2]. Montgomery DW. The cause of the streaks in nevus linearis. J Cutan Genitourinary Dis 1901; 19: 45564.

[3]. Moss C. Cytogenetic and molecular evidence for cutaneous mosaicism:The Ectodermal origin of Blaschko lines. Am J Med Genet 1999; 85: 330-3.

[4]. Jackson R. The lines of Blaschko: a review and reconsideration. Br J Dermatol 1976; 95: 349-60.

[5]. Huh W,Fujiwara K et al. Congenital acantholytic dyskeratotic epidermal nevus following Blaschko's lines versus segmental Darier's disease. Eur J Dermatol. $2007 ; 17(2): 130-2$.
[6]. Lichen Striatus: Clinical features and follow up in 12 patients. Hauber $\mathrm{K}$, Rose $\mathrm{C}$, Brocker EB, Hamm H. European Journal of Dermatology 2000, Oct-Nov; 10(7): 536-9.

[7]. Boyd AS, Neldner KH. Lichen planus. J Am AcadDermatol 1991; 25:593-619.

[8]. LEVER'S Histopathology of skin -David E. Elder -10th edition. Page no. 185-91.

[9]. Rook's Textbook of Dermatology-Tony Burns/Stephen Breathnach, 8th Edition Vol 1;18.4 -18.5, 51-70,

How to cite this article: N. Ramanjanamma, Garnipudi Pavani, Jasmine D. CLINICAL PROFILE OF LINEAR DERMATOSES EMPHASIZING ON THEIR PRESENTATION ALONG THE BLASHCO'S LINES. Int J Intg Med Sci 2018;5(7):687-692. DOI: 10.16965/ijims.2018.124 